\section{Funding hiccup at Jodrell}

\section{Jodrell Bank, Cheshire}

Just as British radioastronomy is coming into its own, funding problems threaten to undermine its work. Britain's leading centre for radioastronomy, the University of Manchester's Nuffield Radio Astronomy Laboratory at Jodrell Bank, expects to find itself short of up to $£ 800,000$, nearly one-third of its annual running cost, when the Universities Funding Council (UFC) this week tells the universities how much money each will get in 1991-92.

Ironically, the problem has appeared just as British radioastronomy is poised to reap the scientific benefits of upgrading its most valued asset - the MERLIN radio telescope array (see this page).

Unlike many of the British laboratories now faced with a declining budget, the problem at Jodrell Bank is not simply the general one of underfunding in the British science base. Jodrell's particular difficulty is the new rigid formula that the UFC is using to calculate the money each university is given for research.

Although the formula does take into account the quality of research in university departments so that more is spent where the research output is strong, the formula will not justify the uniquely large sum of money Manchester must put aside to support radioastronomy at Jodrell. There has always been an element of "special funding" for Jodrell, says its director Rod Davies, but the UFC's predecessor, the University Grants Committee, never gave a detailed breakdown of each university's grant.

The problem is compounded by the government's decision to shift some of the UFC's research money to the research councils, beginning with $£ 50$ million in 1992-93. The idea is to make the research councils responsible for the indirect costs of research they support (see Nature 349, $360 ; 31$ January 1991), but Jodrell staff fear that the transfer will further undermine Manchester's capacity to fund the radioastronomy laboratory.

The obvious solution, Davies says, is for the $£ 800,000$ shortfall to come through the research council system, either from the Science and Engineering Research Council (SERC) or directly from the Advisory Board for the Research Councils (ABRC). SERC is already paying $£ 2.3$ million over three years to run the MERLIN telescope array as a national facility. But Davies says that Jodrell as a whole serves the entire British radioastronomy community, and so deserves increased research council support.

Nevertheless, Davies does not want Jodrell to become one of SERC's own laboratories. Jodrell can be run more efficiently within a university, he says.

Manchester officials saw the problem coming almost a year ago, but their discussions with SERC and the ABRC have so far failed to find a solution. With the research councils, and SERC in particular, tightening their belts to keep their spending within the disappointingly low 1991-92 science budget, Davies finds it difficult to see where the money will come from. The deadlock has also placed in an embarrassing position the SERC chairman, Sir Mark Richmond, as he has changed sides half-way through the negotiations. Until October last year, he was vice-chancellor at Manchester.

Despite the current impasse, Davies is optimistic: "It would be so daft", he says, if the reorganization of university funding could jeopardize a world-famous laboratory. If the money cannot be found, Manchester could be forced to stop funding the Lovell telescope, an instrument that has identified a number of rapidly spinning millisecond pulsars - among the most exciting discoveries by radioastronomers in recent years.

Peter Aldhous

\title{
Making the most of MERLIN's potential
}

\section{Jodrell Bank, Cheshire}

MerLin (the multi-element radio-linked interferometer network) takes advantage of the fact that a number of small radiotelescopes can be made to mimic a single large telescope by making simultaneous observations and combining their data electronically.

This solves a fundamental problem in radioastronomy: a radiotelescope's collection dish must be thousands of times

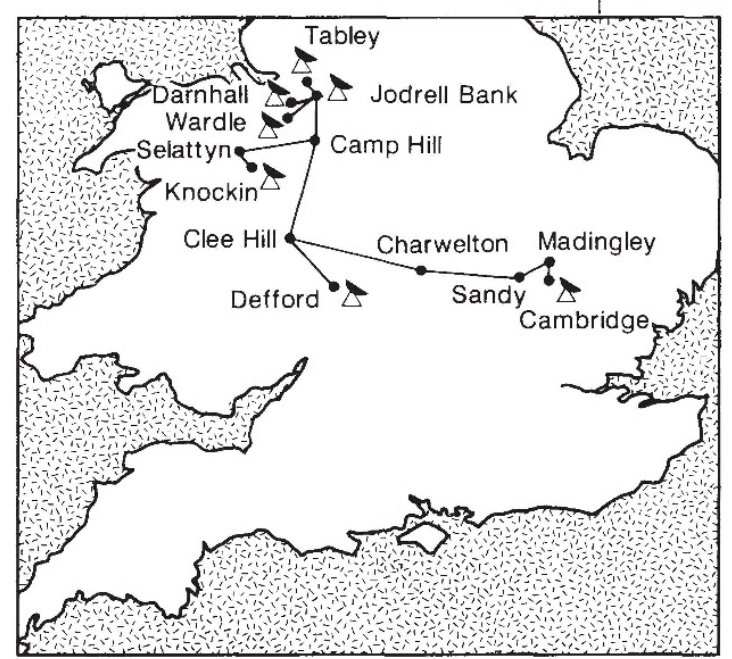
stations showing the microwave links to Jodrell bank.
Map of the MERLIN array of radiotelescopes and repeater

Telescope on the ground", says Rod Davies, Jodrell's director.

The full seven-telescope array should become fully operational in April. Among the first astronomers to benefit will be those interested in the radio observation of thermally active stars. Most stellar radioastronomy has so far concentrated on the few stars that "put on a show" in the radio spectrum, says Dennis Walsh, who collaborated with optical astronomers to discover the first gravitational lens at Jodrell in 1979. MERLIN is now sensitive enough to study "the silent majority" of stars, he says. MERLIN will also be used to investigate the astrophysics of star formation. The interiors of star-forming regions are opaque to optical and infrared telescopes, but can be probed using radioastronomy.

Walsh's own speciality gravitational lenses - is high on the list of projects to be given telescope time. MERLIN will reobserve the first lens to be discovered, enabling astronomers to study the distribution of matter in a galaxy that is 'bending' radio emissions from larger than the mirror of an optical telescope to achieve the same resolution.

Since 1980, six British telescopes have been run together, controlled from Jodrell. But MERLIN's sensitivity has now been increased by fitting the telescopes with helium-cooled radio receivers, improving the microwave links from each back to Jodrell, and upgrading the computers that are used to combine the data.

Even more valuable is a new 32-metre telescope at Cambridge (see Nature 348, $377 ; 29$ November 1990 ) that has increased the maximum distance between pairs of MERLIN telescopes to $\mathbf{2 3 0}$ kilometres, improving MERLIN's angular resolution to better than that of the best ground-based optical telescopes. "It's the Hubble Space a distant quasar to produce a double image. By linking MERLIN's observations with those from other European radiotelescopes, it may be possible to find a third quasar image that is predicted by theory but is likely to be quite faint.

There is also a plan to generate radio emission maps of the sky with MERLIN which will complement the optical maps now being drawn up using data from the European Space Agency's Hipparcos astrometry satellite. Simultaneous observations from very widely spaced radiotelescopes have been used previously to generate radio emission maps, but until now it has not been possible to include the radio emissions from most of the thermally active stars seen by Hipparcos.

Peter Aldhous 